Disponível em

ANEAD http://www.anpad.org.br/rac

RAC, Rio de Janeiro, v. 17, n. 6, art. 6, pp. 739-763, Nov./Dez. 2013

$($ (c) EY-No

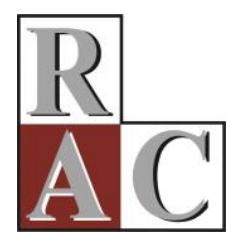

\title{
Rede de Pesquisadores de Finanças no Brasil: Um Mundo Pequeno Feito por Poucos
}

\author{
The Finance Research Network in Brazil: A Small World
}

Wesley Mendes-da-Silva

E-mail: mr.mendesdasilva@gmail.com

Fundação Getulio Vargas - EAESP/FGV

EAESP/FGV, Rua Itapeva, 474, $8^{\circ}$ Andar, 01332-000, São Paulo, SP, Brasil.

Luciana Massaro Onusic

E-mail: lucianaonusic@gmail.com

Universidade Federal de São Paulo - EPPEN/ UNIFESP

EPPEN, Rua Angélica,100, Jardim das Flores, 06110-295, Osasco, SP, Brasil.

Ernesto Michelangelo Giglio

E-mail: ernesto.giglio@gmail.com

Universidade Paulista - UNIP

UNIP, Rua Dr. Bacelar, 1212, Indianópolis, 04026-002, São Paulo, SP, Brasil.

Artigo recebido em 09.06.2013. Última versão recebida em 06.10.2013. Aprovado em 13.10.2013. 


\title{
Resumo
}

O estudo do papel das redes de colaboração na produção de conhecimento é um aspecto relevante, tendo atraído a atenção de uma parcela substancial de pesquisadores e formadores de políticas públicas ao redor do mundo. Este trabalho objetiva analisar as propriedades estruturais das redes de relações entre os pesquisadores na área de Finanças no Brasil. Com base em dados pertencentes a 532 artigos, produzidos por 806 pesquisadores, entre 2003 e 2012, os principais resultados alcançados por meio de Análise de Redes Sociais sugerem que: (a) o ambiente brasileiro possui características estruturais que indicam a existência de Small Worlds; (b) uma pequena parcela $(\sim 3 \%)$ dos pesquisadores apresenta produção com regularidade; (c) quanto maior a centralidade dos pesquisadores na rede, maior a quantidade de artigos por eles publicados.

Palavras-chave: análise de redes sociais; cooperação; inovação em finanças.

\begin{abstract}
The study of the role of collaboration networks in the production of knowledge is important and has attracted the attention of a substantial number of researchers and policy makers around the world. This paper aims to analyze the structural properties of relationship networks among Finance researchers in Brazil. By applying Social Network Analysis to data from 532 articles produced by 806 researchers between 2003 and 2012, this article's results suggest that: (a) the Brazilian environment has structural features that indicate the existence of Small Worlds; (b) a small fraction $(\sim 3 \%)$ of researchers has regular production; (c) the higher the centrality of researchers in the network, the greater the number of articles published by them.
\end{abstract}

Key words: social network analysis; cooperation; financial innovation. 


\section{Introdução}

Um argumento que tem recebido crescente atenção na comunidade de finanças é que as crises exigem soluções inovadoras. Ao mesmo tempo, o processo de inovação está, ao menos em parte, apoiado no arranjo, de pessoas e de instituições, em rede (Chassagnon \& Audran, 2011). Adicionalmente, trabalhos recentemente publicados no Brasil têm apontado a necessidade de pesquisas mais orientadas ao desenvolvimento de tecnologias para a resolução de problemas característicos do mercado nacional (Leal, Almeida, \& Bortolon, 2013).

Assim, assumindo que o processo de inovação em finanças está em grande medida apoiado na condução de pesquisas, não é equívoco supor que o sucesso de propostas inovadoras dependerá da colaboração em pesquisa. Contudo, no Brasil, pouco se sabe a respeito da estrutura das redes de pesquisadores em Finanças, e esse é o foco principal deste trabalho, constituindo-se na principal contribuição desta pesquisa, na medida em que aborda essa relevante matéria (Chan, Chen, \& Fung, 2009; Chan, Chen, \& Lung, 2007; Leal et al., 2013; Lemarchand, 2012). Nesse sentido, em geral, a avaliação de campos científicos tem como unidade de análise os artigos publicados.

A esse respeito, o conhecimento científico, materializado na publicação de tais artigos, é socialmente construído, a partir da cooperação entre pesquisadores no processo de investigação e teorização. E o estudo do relacionamento entre eles pode trazer contribuições significativas para o entendimento da dinâmica do campo de conhecimento (Kuhn, 1978; Merton, 1973). Essa visão moderna tem suportado o surgimento e o incremento de estudos focados no entendimento da estrutura e da dinâmica da colaboração em pesquisas científicas ao redor do mundo (Lemarchand, 2012; Pepe, 2010).

Isso posto, este artigo propõe-se a analisar a estrutura da rede de relacionamento entre os pesquisadores que publicaram nos principais periódicos brasileiros do campo de Finanças, no período de 2003 a 2012. A pesquisa identifica os pesquisadores mais prestigiosos na rede de pesquisadores de finanças no ambiente brasileiro, classificando-os segundo a regularidade de sua produção. Além disso, testa a validade da hipótese de mundos pequenos para a rede de pesquisadores, verificando também a existência de associações entre prestígio do pesquisador e sua produção científica no campo.

$\mathrm{O}$ artigo está organizado em cinco seções, incluindo esta introdução. $\mathrm{Na}$ seção 2, são apresentados elementos teóricos e empíricos da Análise de Redes Sociais (ARS), os quais suportam o presente trabalho. A seção 3 detalha os procedimentos metodológicos adotados no desenvolvimento desta pesquisa. Em seguida, a seção 4 apresenta e discute os resultados da análise da rede de pesquisadores do campo de Finanças no Brasil. Na quinta e última seção, encontram-se as considerações finais.

\section{Bases Teóricas e Empíricas do Estudo}

A questão da produtividade acadêmica nos diversos campos do conhecimento tem sido alvo constante de pesquisas, incluindo a área de Finanças, com predomínio dos temas sobre as dificuldades e facilitadores da produção de conhecimento na forma de artigos científicos. Nesse sentido, Chan, Chen e Fung (2009) examinaram os efeitos do pedigree and placement sobre a produtividade em finanças, e encontraram resultados que sugerem que pesquisadores ligados a instituições de elevada reputação (por eles chamadas de instituições de elite) tendem a ser mais produtivos, destacadamente quando considerados os journals de maior impacto. 


\section{Colaboração na produção científica em finanças}

O estudo de questões associadas aos trabalhos publicados em periódicos especializados em temas de Finanças é um tema já existente na literatura (Oltheten, Theoharakis, \& Travlos, 2005). Chan, Chen e Lung (2007) estudaram resultados de pesquisas de alcance internacional em Finanças, publicados no período de 1990-2004. Outras pesquisas mais recentes têm dedicado atenção ao entendimento de padrões dos trabalhos publicados no campo de Finanças, tal como Hardin, Liano, Chan e Fok (2008), os quais analisaram o impacto da participação em conselhos editoriais de revistas científicas sobre a produtividade em pesquisa.

Investigar a produção de um campo tem importância, já que pode revelar: (a) as tendências temáticas; (b) os centros universitários mais produtivos; (c) os autores mais proeminentes; (d) os artigos mais citados; (e) as questões de pesquisa repetidas; e (f) caminhos futuros de pesquisa. Um dos usos possíveis desses trabalhos está em desempenhar o papel de guia para a construção e a proposição de temas e problemas em novas pesquisas de fronteira, na medida em que se busca entender a rede de autores, foco desta pesquisa.

Então, um dos pontos de interesse nesses trabalhos, de cunho bibliográfico, é investigar a rede de coautores do campo de finanças. Ressalte-se que a premissa central do presente estudo é que o conhecimento é socialmente construído, e que, por extensão: a promoção da produção no campo de Finanças está vinculada ao entendimento da rede de pesquisadores que nele atuam (Chan, Chen, \& Steiner, 2004).

\section{Análise de redes sociais}

Conforme Castells e Cardoso (2005) e Nohria e Ecles (1992), a sociedade está organizada na forma de redes e a investigação das instituições, sejam de negócios, ou de políticas públicas, ou de outra natureza; podem ser analisadas na perspectiva das redes. A produção acadêmica sobre redes está organizada em torno de dois paradigmas principais: o racional-econômico e o social. O paradigma racional-econômico assume que as redes são construções planejadas, principalmente para a solução de dependência de recursos e de custos (Gulati \& Gargiulo, 1999).

O paradigma social, foco deste trabalho, assume que as redes se formam a partir do contexto das relações sociais. Isso inclui as relações de aproximação entre os atores (com experiências de confiança e comprometimento) e as relações de distanciamento (com as experiências de competição e jogos de poder), tal como detalha Granovetter (1985). Dito de outra forma, duas ou mais pessoas tendem a trabalhar em conjunto quando existem laços sociais entre elas. Esta perspectiva de análise das relações sociais entre atores de uma rede é um campo de muitas expressões, entre elas a ARS, que, por sua vez, deve ser entendida em duas perspectivas basilares.

A primeira perspectiva da ARS é a teórica, e a segunda é a metodológica. A perspectiva teórica segue o paradigma social das teorias de redes. Ou seja, assume o princípio de que existem relações sociais entre atores, além das relações de cunho racional e econômico. Já na perspectiva metodológica, designa-se um conjunto de técnicas de análise das relações, as quais buscam analisar a estrutura da rede e a posição dos atores que dela fazem parte. A partir do entendimento da estrutura da rede e do posicionamento dos atores, é possível discutir questões de explícita relevância, e.g. poder, clusters e liderança, entre outros. Entre os conceitos de ARS que interessam aos propósitos do presente trabalho (adiante discutidos), ressalta-se: a centralidade e a eficiência dos laços constituídos pelos pesquisadores, a densidade e a configuração de clusters por eles formados.

\section{Densidade}

A densidade $(\Delta)$ é um parâmetro relativo à configuração geral da rede, que expressa a relação entre o número de laços constituídos ( $l$ ) no âmbito de um grupo de atores e o número de laços que poderiam ser constituídos no âmbito dessa rede (Knoke \& Kuklinski, 1982). Esse indicador estrutural 
da rede varia no intervalo $[0,1]$, quanto mais próximo $\Delta$ for de zero, menos conectada é a rede. De forma alternativa, as redes cujo valor para $\Delta$ estiverem mais próximos de 1 , serão mais conectadas, tal como apresentado em (1).

$$
\Delta=\frac{l}{n(n-1) / 2}
$$

Em ambientes de alta densidade de relações, o conteúdo da rede, i.e., os laços constituídos por um ator, pode se tornar redundante (Kogut \& Walker, 2001). De forma alternativa, redes de baixa densidade podem ser caracterizadas por laços fracos, i.e., de baixa redundância ou mais eficientes (Burt, 1992). A esse respeito, Kuhn (1978) aponta que novas formas de pensamento podem ser vistas como inconsistentes, se acaso considerar-se comunidades científicas altamente coesas (mais densas, e, portanto redundantes). Assim, interações com outros pesquisadores, externos ao seu grupo, são vistas como relevantes.

\section{Clusters}

Conforme Wasserman e Faust (1994) e Scott (2000), uma rede pode conter diversos subgrupos, chamados componentes da rede. Componentes constituem clusters com alta densidade interna e fraca densidade externa. Os clusters podem existir por vários motivos, entre os quais o compartilhamento de interesses, expectativas e valores éticos comuns e conhecimentos semelhantes. Então, o componente principal será a sub-rede totalmente conectada, e que contempla a maior quantidade de atores no contexto da rede.

\section{Centralidade}

Centralidade é uma propriedade que reflete o quão central um determinado ator $i$ é na rede da qual ele faz parte. É frequente, em ARS, atribuir à identificação dos atores mais centrais da rede a relevância de seu posicionamento no contexto desta. Existem diversas proxies para estimar a centralidade de um ator no âmbito de uma rede. Entre essas, as mais populares são: (a) centralidade de grau (Degree); e (b) centralidade de intermediação (Betweenness), conforme advogam Scott (2000) e Wasserman e Faust (1994). Em termos formais, Degree é o número de laços que um ator $i$ constitui com outros atores a ele adjacentes (Wasserman \& Faust, 1994). Segundo Scott (2000), Degree leva em consideração apenas as relações adjacentes, mostrando a centralidade local do ator $i$. Em termos absolutos, um ator inserido em uma rede composta por $g$ atores pode alcançar, no máximo, $(g-1)$ laços. Segundo Freeman (1978), o índice de centralidade de grau, definido por $C_{D}\left(n_{i}\right)$ de um ator $n_{i}$, participante de uma rede, é dado por (2).

$$
C_{D}\left(n_{i}\right)=d\left(n_{i}\right)=x_{i+}=\sum_{j} x_{i j}=\sum_{j} x_{j i}
$$

Contudo, interações entre dois atores não adjacentes podem depender de um conjunto de outros atores, os quais podem exercer algum controle sobre as interações entre dois atores não adjacentes. Desse modo, se para colocar em contato dois atores $n_{2}$ e $n_{3}$, o menor caminho é $n_{2} \rightarrow n_{1} \rightarrow n_{4} \rightarrow n_{3}$, então, pode-se dizer que os atores $n_{1}$ e $n_{4}$ controlam as interações entre os atores $n_{2}$ e $n_{3}$. Trata-se, portanto, do conceito de Betweenness que contempla a interação entre atores não adjacentes. Nesse sentido, conforme argumentos de Freeman (1978) e de Wasserman e Faust (1994), um ator é um agente se ele está conectado a vários outros atores não diretamente conectados entre si. Uma ideia formal para o conceito de Betweenness é apresentada por Pitts (1979), o qual supõe que, se um ator $j$ deseja contatar um ator $k$, então, um ator $i$ necessita ser usado como uma estação de intermediação.

Então, o ator $i$, possui certa responsabilidade na rede de relacionamentos para com os atores $j \mathrm{e}$ $k$. Desse modo, se forem contadas todas as comunicações de distância $d\left(n_{j}, n_{k}\right)$ as quais passam através do ator $i$, tem-se uma medida de stress. Quando existe mais de um caminho possível entre $j$ e 
$k$, todos os caminhos que passam pelo ator $i$ são considerados equiprováveis. Dessa forma, Betweenness para $n_{i}$ é a soma das probabilidades estimadas para todos os pares de atores não se incluindo o $i$-ésimo ator, e é dada por (3):

$$
C_{B}\left(n_{i}\right)=\sum_{j<k} \frac{g_{j k}\left(n_{i}\right)}{g_{j k}}
$$

em que $g_{j k}$ é o número de caminhos que ligam dois atores. Assim, se todos esses caminhos são equiprováveis para escolha para estabelecer comunicação, a probabilidade de um caminho ser escolhido é simplesmente $\frac{1}{g_{j k}}$. Contudo, Freeman (1978) considera a possibilidade de ator $i$ distinto estar envolvido na comunicação entre dois atores, a qual contém o ator $i$. Nessa situação, a probabilidade seria dada por $\frac{g_{j k}\left(n_{i}\right)}{g_{j k}}$, assumindo a premissa de que os caminhos são equiprováveis para escolha no estabelecimento da comunicação.

\section{Structural holes}

Se pesquisadores individualmente se beneficiam da colaboração para ter maior produtividade (Lee \& Bozeman, 2005), espera-se que aqueles mais centrais apresentem maior probabilidade de possuírem maior produtividade. Além da quantidade de laços diretos desenvolvidos por cada autor (i.e. centralidade de grau), a eficiência destes laços permite avaliar como as lacunas estruturais (Structural Holes) da rede são exploradas (Burt, 1992). Pressupõe-se que, quanto maior a diversidade dos laços de um indivíduo na rede, maior o acesso a recursos diferentes do grupo social que interage com maior frequência (Lin, 2001; Mizruchi, 1996). Assim, para Burt (1992), os Structural Holes são caracterizados pelos relacionamentos não redundantes entre dois atores. Quanto menor o número de laços redundantes, maior o número de lacunas estruturais na rede, havendo menor redundância de informação (Borgatti, 1997). O elemento fundamental das lacunas estruturais está na extensão que a estrutura social de uma arena competitiva cria oportunidades para certos agentes, por meio de seus relacionamentos. Nesses termos, o controle e a participação na difusão da informação definem o capital social das lacunas estruturais (Burt, 1992).

Diante desses argumentos, pode-se assumir o seguinte raciocínio: se laços não redundantes (i.e. eficientes) permitem a determinado pesquisador participar de diferentes grupos e possibilitam o acesso a diferentes recursos, espera-se que esse pesquisador potencialize sua produção de trabalhos científicos. Por um lado, isso significa que as lacunas estruturais aumentem as suas chances de produzir maior número de artigos em coautoria com outros pesquisadores com habilidades complementares à sua. Por outro, isso indica que, se há chances do conhecimento e dos recursos compartilhados a partir dos laços não redundantes serem uma fonte de vantagens para o pesquisador com maior proporção desses laços, espera-se que indivíduos que consigam desenvolver número maior de laços não redundantes sejam mais produtivos que os demais.

\section{Small worlds}

De acordo com Watts e Strogatz (1998) e Watts (1999), Small Worlds ocorrem quando atores participantes de uma rede estão agrupados de forma esparsa, mas, ao mesmo tempo, eles estão conectados a atores externos ao seu grupo, por meio de um pequeno grupo de atores intermediários. Do ponto de vista teórico, o conceito de Small Worlds está próximo da abordagem de análise de coesão (Coleman, 1990), Structural Holes (Burt, 1992) ou laços fracos (Granovetter, 1973). Ao mesmo tempo em que existem ligações com outros grupos, nas quais a informação é não redundante (i.e. mais eficiente), existe um nível de coesão das atividades necessárias para se tornar conhecido entre os membros da rede (Uzzi \& Spiro, 2005). Assim, as propriedades de um mundo pequeno produzem elementos ligados à durabilidade das estruturas de relacionamento, tais como autores e instituições, essenciais para a compreensão da mútua relação entre as estruturas locais e globais. 
O potencial da ARS na investigação da colaboração científica vem sendo explorado por estudos recentes (Barabási et al., 2002; Moody, 2004; Newman, 2001). Estes trabalhos evidenciam o crescimento da cooperação entre os pesquisadores, cujas relações não só aumentam em frequência, mas também em número de colaboradores, possibilitando a formação de redes. Assim, a avaliação da construção do conhecimento científico pode partir de uma nova perspectiva: o conjunto de relacionamentos entre pesquisadores. É comum que estudos deste tipo considerem como base analítica a cooperação entre cientistas em termos de coautoria - i.e., participação de dois ou mais autores na produção de um estudo - por se configurar como uma manifestação formal de colaboração intelectual na pesquisa científica (Acedo, Barroso, Casanueva, \& Galan, 2006; Moed, Glänzel, \& Schubert, 2005).

Devido à intermediação de alguns atores numa rede, os grupos podem não estar isolados, mas conectados entre si, sendo que os laços por eles estabelecidos possibilitam a comunicação entre grupos distintos. Dessa forma, as propriedades de Small Worlds propiciam elementos para análise da durabilidade das estruturas de relacionamento, bem como de práticas e valores científicos (Kogut \& Walker, 2001), o que é importante para o melhor entendimento da relação entre estruturas locais e globais da rede. Estruturas de rede do tipo Small World foram verificadas nas áreas de Física por Newman (2001), Sociologia por Moody (2004), e Economia por Goyal, Van der Leij e MoragaGonzález (2004). No contexto brasileiro, resultado semelhante foi encontrado por Rossoni e Machadoda-Silva (2008) na área de Estudos Organizacionais e Estratégia, e por Martins, Rossoni, Csillag, Martins e Pereira (2010) no campo de Gestão de Operações.

O modelo teórico de Small Worlds é descrito em detalhes por Newman, Strogatz e Watts (2001). Duas características (globais da rede) desse modelo teórico, o qual exerce fortes consequências para o fenômeno social, devem ser observadas com atenção destacada na caracterização de Small Worlds: (a) a distância entre os atores $(L)$; e (b) valores elevados para coeficiente de agrupamento, ou cluster $\left(C_{\Delta}\right)$. À luz das métricas encontradas, a constatação rigorosamente típica de Small World será verificada se $L_{\text {Observado }} \sim L_{\text {Esperado, }}$, enquanto $C_{\Delta \text { observado }} \gg C_{\Delta_{\text {Esperado }}}$. Contudo, tal como procedimento assumido por Baum, Rowley e Shipilov (2004) e Davis, Yoo e Baker (2003), nesta pesquisa a verificação da existência de Small Worlds no contexto das redes de pesquisadores de finanças será satisfeita quando $\Theta=\left(C_{\Delta \text { observado }} / L_{\text {Observado }}\right) \times\left(L_{\text {Esperado }} / C_{\Delta_{\text {Esperado }}}\right) \gg 1$.

\section{Procedimento}

\section{Coleta de dados}

Os dados empregados na presente pesquisa são oriundos de periódicos científicos brasileiros. Esse conjunto inclui 11 revistas selecionadas mediante critérios adotados por Leal, Almeida e Bortolon (2013), adicionando-se a revista Brazilian Business Review (BBR), classificada como A2 no Qualis/Capes no momento do desenvolvimento deste estudo. Assim, os periódicos escolhidos foram os que se encontram listados na Tabela 1. Desse modo, os dados empregados neste trabalho refletem um conjunto total de 532 artigos publicados no campo de Finanças, por 806 autores, em 12 diferentes revistas científicas brasileiras no período 2003-2012.

\section{Ferramentas de análise}

A análise foi conduzida a partir de três momentos distintos: (a) identificação dos autores mais centrais e prolíficos; (b) verificação da existência de Small Worlds na rede de pesquisadores de finanças; (c) verificação da existência de associações entre posicionamento do pesquisador na rede e sua produção, por meio de regressões OLS. Com a intenção de classificar os autores em relação à sua presença no campo, utilizou-se o procedimento proposto por Braun, Glänzel e Schubert (2001). 
Tabela 1

Distribuição dos Artigos de Finanças a partir de Revistas Brasileiras Selecionadas (Período de 2003 a 2012)

\begin{tabular}{lccccccccccc}
\hline Revistas & 2003 & 2004 & 2005 & 2006 & 2007 & 2008 & 2009 & 2010 & 2011 & 2012 & Total \\
\hline RBFin & 10 & 9 & 10 & 10 & 10 & 15 & 19 & 20 & 22 & 23 & 148 \\
RAUSP & 7 & 12 & 7 & 7 & 15 & 6 & 6 & 3 & 4 & 3 & 70 \\
RC\&F & 3 & 1 & 6 & 12 & 8 & 7 & 7 & 4 & 5 & 4 & 57 \\
BBR & - & 2 & 1 & 2 & 6 & 10 & 6 & 5 & 7 & 5 & 44 \\
RAM & 1 & & 1 & 4 & 2 & 2 & 10 & 7 & 6 & 10 & 43 \\
RAC & 4 & 3 & 1 & 6 & 2 & 6 & 3 & 4 & 5 & 4 & 38 \\
G\&P & - & 2 & 3 & 1 & 2 & 3 & 6 & 9 & 3 & 6 & 35 \\
PROD & 3 & 1 & - & 3 & - & 3 & 1 & 2 & 8 & 4 & 25 \\
RAE & 2 & 3 & 1 & 1 & - & 5 & 3 & 1 & 2 & 3 & 21 \\
RBE & 3 & 3 & 2 & 1 & 2 & - & 3 & 2 & - & 4 & 20 \\
EA & - & - & 1 & 3 & 1 & - & - & 3 & 4 & 4 & 16 \\
BAR & - & - & 1 & - & - & 3 & - & 2 & 3 & 6 & 15 \\
Total & 33 & 36 & 34 & 50 & 48 & 60 & 64 & 62 & 69 & 76 & 532 \\
\hline
\end{tabular}

Nota. Fonte: Elaborado pelos autores com base nos dados coletados. BAR = Brazilian Administration Review; EA = Economia Aplicada; G\&P = Gestão \& Produção; RBE = Revista Brasileira de Economia; RBFin= Revista Brasileira de Finanças; $\mathrm{RC} \& \mathrm{~F}=$ Revista Contabilidade \& Finanças; RAC = Revista de Administração Contemporânea, não foi considerada a RAC Eletrônica; RAM = Revista de Administração Mackenzie; RAUSP = Revista de Administração da Universidade de São Paulo; RAE = Revista de Administração de Empresas, não foi considerada a RAE Eletrônica; PROD = Revista de Produção.

Nessa classificação, os pesquisadores foram categorizados como entrantes, One-timers, Transientes, Continuantes ou Retirantes em função do volume e da regularidade de sua publicação na área ao longo de todo o período investigado. Para tanto, considerou-se: (a) o número de artigos publicados por um pesquisador; (b) o momento em que sua produção ocorreu; e (c) a distribuição da produção ao longo dos anos.

- Continuantes: os pesquisadores que publicaram em 5 ou mais diferentes anos do período em estudo, com intervalo de não mais do que dois anos entre cada publicação e pelo menos uma nos últimos três anos.

- Transientes: possuem mais de uma publicação distribuídas ao longo do período analisado em não mais do que quatro anos diferentes, sendo ao menos uma nos últimos três anos.

- Entrantes: apresentam mais de uma publicação, exclusivamente, em um ou mais anos diferentes dos últimos três anos do período em estudo.

. One-timers: possuem apenas uma única publicação em todo o período analisado.

- Retirantes: são os pesquisadores com mais de uma publicação em um ou mais diferentes anos, mas sem publicações nos últimos três anos. 


\section{Modelo empírico}

Com a intenção de testar a existência de associações entre posicionamento do $i$-ésimo pesquisador na rede e sua produção, foi testado o modelo de regressão expresso em (4).

$$
\text { Produção }_{i}=\beta_{0}+\beta_{1} \cdot \text { Degree }_{i}+\beta_{2} \cdot \text { Betweenness }_{i}+\beta_{3} \cdot \text { Structural Holes }_{i}+\varepsilon_{i}
$$

Em que as variáveis independentes são as duas proxies de centralidade e a métrica de eficiência de laços constituídos pelo pesquisador.

\section{Resultados}

Esta seção, inicialmente, a título de ilustração e exploração, apresenta a evolução do campo de finanças no Brasil, em termos de número de autores, número de artigos e coautorias. Além disso, descreve a evolução de métricas de configuração da rede de autores ao longo do período estudado (2003-2012). Posteriormente, são conduzidos testes para verificação da validade da hipótese de mundos pequenos, mediante procedimento de Newman (2001) e Moody (2004). Finalmente, conforme argumentos defendidos por Acedo, Barroso, Casanueva e Galan (2006) é verificada a existência de associações entre o prestígio social dos pesquisadores, medido pela sua centralidade no âmbito da rede, e sua produção científica no campo de finanças no Brasil.

A respeito da evolução dos indicadores de produção, produtividade e cooperação do campo de Finanças no Brasil, os resultados obtidos sugerem que o número de publicações mais que dobrou na última década, como pode ser observado na Figura 1. Em 2003, 33 artigos foram publicados no campo; em 2012, esse número foi de 76, sugerindo um crescimento anual de $\sim 9,5 \%$. É possível também verificar que o campo de Finanças não cresceu apenas em termos de número de artigos publicados, mas especialmente na quantidade de pesquisadores. Isso ocasionou o crescimento do número de autorias (a cada participação que um autor é encontrado, considera-se uma autoria diferente).

Tal como têm documentado em outros campos, Newman (2001), Barabási et al. (2002) e Moody (2004), neste trabalho observa-se o crescimento da cooperação entre pesquisadores. A partir de 2005, houve certo descolamento entre o volume de autorias, e o número de autores que produziam no campo. Isso denota aumento da produtividade dos pesquisadores, pois indica crescimento do número médio de autorias por autor. 


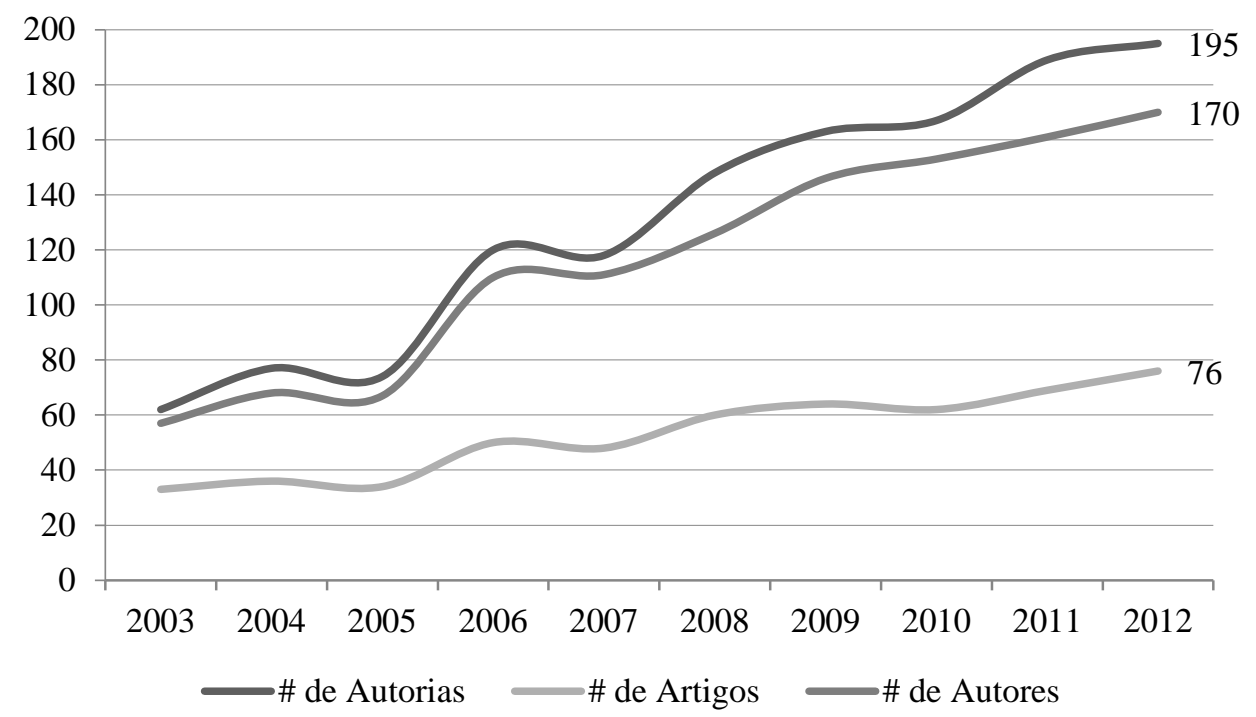

Figura 1. Evolução do Número de Autorias, Artigos e Autores do Campo de Finanças em Revistas Brasileiras.

Fonte: Elaborado pelos autores com base nos dados coletados. Um mesmo autor pode ter sido coautor de mais de artigo, a cada participação considera-se uma autoria diferente para o mesmo autor. Assim, em 2012, 170 autores produziram 76 artigos, os quais ocasionaram 195 autorias.

Entre os autores que mais contribuíram para a literatura financeira publicada no Brasil, conforme ilustra a Tabela 2, encontram-se: Medeiros, O. R.- UnB, com 16 artigos publicados, tendo construído 13 laços diretos decorrentes de parcerias de pesquisa, seguido de Saito, R.- FGV/EAESP, com 13 artigos e 9 laços diretos e Famá, R. - PUC/SP, com 12 artigos e 14 laços diretos. Esses pesquisadores lideram o conjunto de pesquisadores mais prolíficos no campo, no período analisado, 2003-2012. Ainda na Tabela 2, é possível notar que, entre os 26 autores apontados como os mais produtivos no período, três Instituições se destacam.

Tabela 2

Decomposição da Produção dos Autores mais Prolíficos no Campo de Finanças em Revistas Brasileiras (2003-2012)

\begin{tabular}{llcccc}
\hline Autores & Instituição & \# de Artigos & Degree & Betweenness & $\begin{array}{c}\text { Structural } \\
\text { Holes }\end{array}$ \\
\hline Medeiros, O.R. ${ }^{\dagger \dagger}$ & UnB & 16 & 13 & $3.790,65$ & 0,806 \\
Saito, R. ${ }^{\dagger}$ & FGV/EAESP & 13 & 9 & $4.849,24$ & 0,800 \\
Fama, R. $^{\dagger}$ & PUC/SP & 12 & 14 & $8.019,21$ & 0,787 \\
Sheng, H.H. ${ }^{\dagger}$ & FGV/EAESP & 11 & 12 & $4.704,03$ & 0,799 \\
Silveira, A.D.M. ${ }^{\dagger}$ & FEA/USP & 10 & 9 & $6.874,76$ & 0,640 \\
Pinto, A.C.F. & PUC/Rio & 10 & 15 & $2.551,06$ & 0,727 \\
Bressan, A.A. ${ }^{\dagger}$ & UFMG & 10 & 11 & 171,33 & 0,694 \\
Kayo, E.K. ${ }^{\dagger}$ & FEA/USP & 10 & 14 & $3.341,04$ & 0,724 \\
Lopes, A.B. & FEA/USP & 9 & 14 & $5.936,95$ & 0,733 \\
Galdi, F.C. & Fucape & 9 & 13 & $4.857,95$ & 0,735 \\
\hline
\end{tabular}


Tabela 2 (continuação)

\begin{tabular}{|c|c|c|c|c|c|}
\hline Autores & Instituição & \# de Artigos & Degree & Betweenness & $\begin{array}{c}\text { Structural } \\
\text { Holes }\end{array}$ \\
\hline Procianoy, J.L. ${ }^{\dagger}$ & UFRGS & 9 & 8 & 104,00 & 0,778 \\
\hline Mendes-da-Silva, $\mathrm{W} .^{\dagger}$ & FGV/EAESP & 9 & 16 & $4.066,45$ & 0,765 \\
\hline Nakamura, W.T. ${ }^{\dagger}$ & Mackenzie & 9 & 17 & $1.848,35$ & 0,728 \\
\hline Barbedo, C.H.S. ${ }^{\dagger}$ & Ibmec/Rio & 8 & 14 & $4.207,00$ & 0,769 \\
\hline Barros, L.A.B. C. & FEA/USP & 8 & 8 & $4.393,03$ & 0,556 \\
\hline Costa Junior, N.C. A. ${ }^{\dagger}$ & UFSC & 8 & 13 & $2.776,76$ & 0,786 \\
\hline Leal, R.P.C.* & Coppead/UFRJ & 8 & 11 & $9.303,02$ & 0,750 \\
\hline Martinez, A.L. ${ }^{\dagger}$ & Fucape & 7 & 6 & $1.539,69$ & 0,592 \\
\hline Tabak, B.M. & Bacen & 7 & 8 & 26,00 & 0,753 \\
\hline Perobelli, F.F.C. ${ }^{\dagger}$ & UFJF & 7 & 10 & $4.008,43$ & 0,702 \\
\hline Amaral, H.F. ${ }^{\dagger}$ & UFMG & 7 & 11 & 123,83 & 0,708 \\
\hline Brandao, L.E.T. & PUC/Rio & 7 & 10 & $4.855,34$ & 0,702 \\
\hline Corrar, L.J. ${ }^{\dagger}$ & FEA/USP & 7 & 13 & $5.915,29$ & 0,735 \\
\hline Klotzle, M.C. & PUC/Rio & 7 & 12 & $2.209,70$ & 0,645 \\
\hline Pimenta Junior, T. ${ }^{\dagger}$ & FEARP/USP & 7 & 8 & 35,50 & 0,704 \\
\hline Ness Junior, W.L. ${ }^{\dagger}$ & PUC/Rio & 7 & 12 & $2.468,75$ & 0,680 \\
\hline
\end{tabular}

Nota Fonte: Elaborado pelos autores com base nos dados coletados. Esta tabela apresenta os 26 autores mais presentes no campo de Finanças delineado pelas revistas brasileiras, estão reportados dados relativos aos pesquisadores com pelo menos 7 artigos no período estudado. ${ }^{*}$ Pesquisador Bolsista de Produtividade pelo CNPq (em maio de 2013), segundo registros no currículo disponível na plataforma Lattes do CNPq. ${ }^{\dagger}$ Pesquisador classificado como Continuante, mediante critérios propostos por Braun, T., Glänzel, W., \& Schubert, A. (2001). Publication and cooperation patterns of the authors of neuroscience journals. Scientometrics, 50(3), 499-510. doi: 10.1023/A:1012774206340

A FEA/USP, com $5(\sim 19 \%)$ pesquisadores; PUC/Rio, com $4(\sim 15 \%)$ pesquisadores; e FGV/EAESP, com $3(\sim 12 \%)$ pesquisadores. A Tabela 3 apresenta a evolução do número de artigos, autores e autorias, conforme ilustrado anteriormente pela Figura 1. Tais medidas foram utilizadas para calcular os indicadores de cooperação e produtividade dos autores do campo. A cooperação, que indica o número médio de autorias por artigo em cada ano, experimentou incremento de $\sim 36,56 \%$ entre 2003 e 2012.

O número de artigos publicados e de autores atuantes no campo cresceu exponencialmente. A produtividade total, que reflete o número médio de autorias de cada autor também cresceu cerca de $5,42 \%$ no período estudado. Já a produtividade fracionada, que aponta a contribuição média de cada autor por artigo publicado caiu $\sim 22,8 \%$. Assim, apesar de o número de artigos publicados em cada ano, no campo, ter crescido consideravelmente, i.e., 130,3\% entre 2003 e 2012, a produtividade média de cada autor individualmente decresceu no período analisado. É possível constatar que há mais autorias por artigos, o que indica tendência de os pesquisadores terem maior cooperação para a produção de artigos. A subseção a seguir discute a estrutura da rede de cooperação científica no campo de Finanças no Brasil. 
Tabela 3

Evolução das Medidas de Cooperação e Produtividade do Campo de Finanças em Revistas Brasileiras

\begin{tabular}{ccccccc}
\cline { 5 - 6 } & & & & & \multicolumn{2}{c}{ Produtividade no campo $^{\text {Ano }}$} \\
\hline \# de Autorias & \# de Artigos & \# de Autores & Cooperaçãa $^{\mathrm{a}}$ & Total $^{\mathrm{b}}$ & Fracionada $^{\mathrm{c}}$ \\
\hline 2003 & 62 & 33 & 57 & 1,879 & 1,088 & 0,579 \\
2004 & 77 & 36 & 68 & 2,139 & 1,132 & 0,529 \\
2005 & 74 & 34 & 67 & 2,176 & 1,104 & 0,507 \\
2006 & 120 & 50 & 110 & 2,400 & 1,091 & 0,455 \\
2007 & 118 & 48 & 111 & 2,458 & 1,063 & 0,432 \\
2009 & 148 & 60 & 126 & 2,467 & 1,175 & 0,476 \\
2010 & 163 & 64 & 146 & 2,547 & 1,116 & 0,438 \\
2011 & 167 & 62 & 153 & 2,694 & 1,092 & 0,405 \\
2012 & 189 & 69 & 161 & 2,739 & 1,174 & 0,429 \\
\hline
\end{tabular}

Nota. Fonte: Cálculos dos autores a partir dos dados coletados.

autorias/artigo; ${ }^{\mathrm{b}}$ autorias/autores; ${ }^{\mathrm{c}}$ artigos/autores.

\section{Rede de cooperação dos pesquisadores do campo de Finanças}

Nesta seção, são expostos os resultados do estudo longitudinal dos relacionamentos entre os pesquisadores do campo de Finanças no Brasil, no período 2003-2012. Inicialmente, é analisada a estrutura da rede de cooperação dos autores no campo ao longo do período, e analisa-se a evolução das medidas estruturais da rede e das medidas de relacionamento, bem como a existência da dinâmica de mundos pequenos (Small Worlds). A Figura 2 representa a rede de pesquisadores para todo o período de análise (2003-2012), de forma agregada.

Nessa figura, é possível observar cores diferentes para os nós presentes na rede, i.e., os pesquisadores. Os nós na cor negra representam o componente principal, sendo este uma sub-rede em que os nós estão conectados entre si (Nooy, Mrvar, \& Batagelj, 2005; Wasserman \& Faust, 1994). A partir da identificação de um grande número de cores, verifica-se a existência de um grande número de autores que não cooperam diretamente, ou mesmo indiretamente, entre si. A Figura 2 apresenta a evolução da configuração da rede de pesquisadores entre os três subperíodos adotados, nela fica ilustrado o crescimento da rede de pesquisadores. A existência de vários grupos de autores, i.e., componentes ou clusters, indica fragmentação do campo de Finanças. Conforme Moody (2004), quando em redes de colaboração, os pesquisadores tendem a possuir maior facilidade de compartilhar ideias, de tal maneira que um pesquisador possa influenciar a atividade científica do outro. 


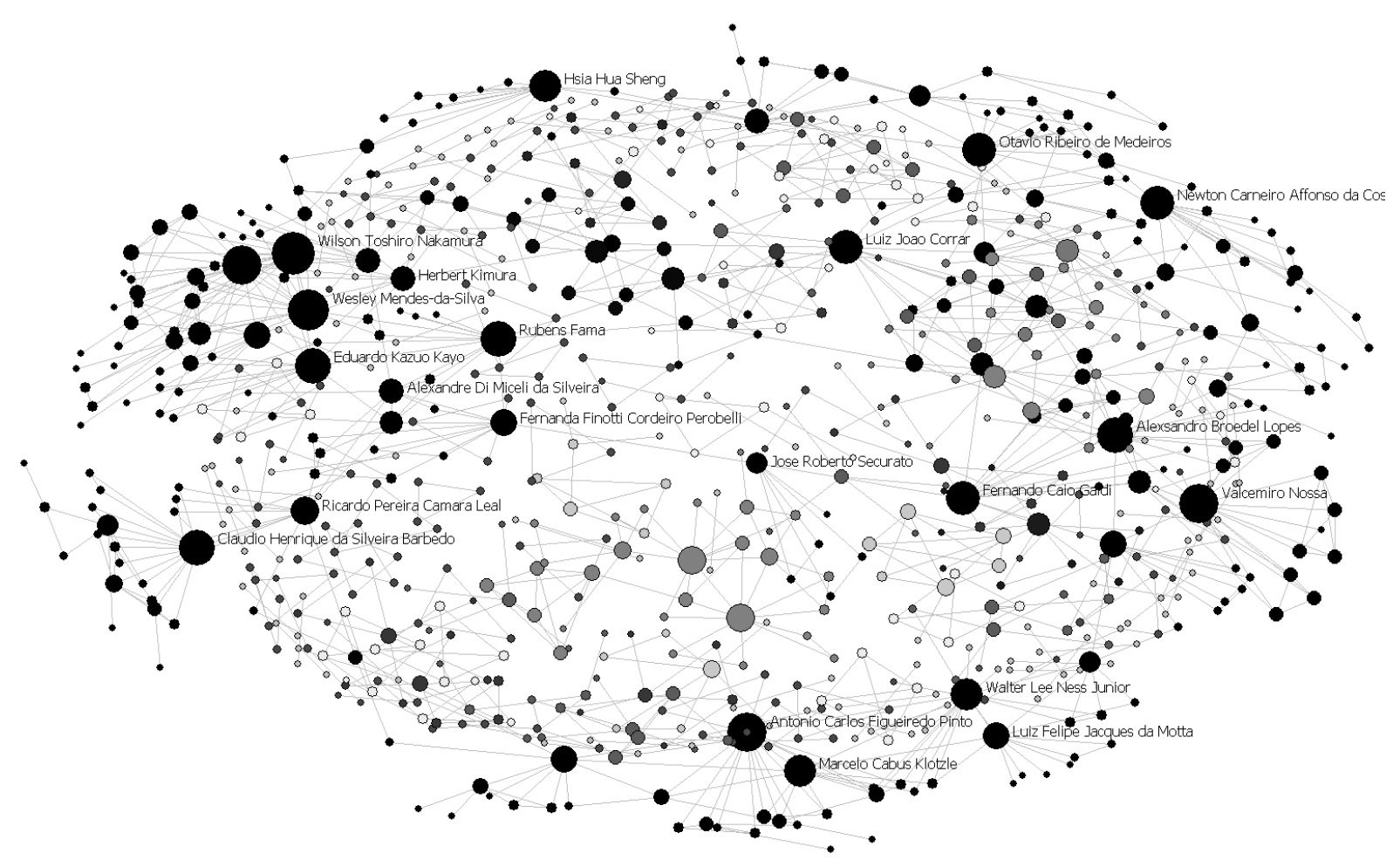

Figura 2. Estrutura da Rede de Colaboração entre Pesquisadores do Campo de Finanças (2003-2012).

Fonte: Elaborado pelos autores com base nos dados coletados. Cada uma das diferentes cores dos nós indica um dos 182 componentes da rede, i.e., agrupamento de pesquisadores ligados entre si. O conjunto de nós na cor negra indica a configuração do componente principal da rede de pesquisadores de Finanças, onde estão 260, dos 806 pesquisadores. Não estão representados na figura os 33 autores isolados, i.e., aqueles que produziram artigos sem que tenha sido estabelecida alguma parceria de pesquisa.

A esse respeito, a Tabela 4 apresenta as estatísticas descritivas das estruturas de relacionamentos entre os autores e sua evolução nos três subperíodos analisados. A última coluna, à direita, que traz o agregado dos três subperíodos (2003-2012), apresenta a rede de relacionamentos de autores, de forma agregada. Ao constatar que o número de autorias cresceu em maior ordem que o volume de artigos, indicando mais autorias por artigo e, consequentemente, maior relacionamento entre os autores, tal relacionamento reflete-se no número médio de laços por autor (número de autores com que cada autor colaborou, onde não se considera o número de vezes que colaborou, conforme estabelecem Nooy, et al., 2005), demonstrando uma estrutura de colaboração. Segundo Nooy, Mrvar e Batagelj (2005), quando há queda no ritmo de crescimento da rede de relacionamentos ao longo dos períodos, há indicação de amadurecimento da estrutura da rede. A partir desse argumento, nota-se que o campo de Finanças no Brasil ainda pode ser visto como pouco maduro, tendo em vista o explícito crescimento obtido nos últimos anos (ver Figura 1 e Tabela 3). Com base no conteúdo da 4, observa-se que, em termos relativos, a proporção de autores que publicavam isolados caiu ao longo do período, de $\sim 8 \%$ no primeiro subperíodo para $\sim 2 \%$ do total de autores no último. 
Tabela 4

Estatísticas Descritivas da Estrutura da Rede de Pesquisadores em Finanças no Brasil

\begin{tabular}{lcccc}
\hline & Período \#1 & Período \#2 & Período \#3 & Agregado \\
\cline { 2 - 4 } Parâmetros & $2003-2006$ & $2007-2009$ & $2010-2012$ & $2003-2012$ \\
\hline Artigos & 153 & 172 & 207 & 532 \\
Autores & 255 & 317 & 410 & 806 \\
Autorias & 333 & 429 & 551 & 1.313 \\
Média de Laços por Autor & 1,812 & 2,341 & 2,341 & 2,551 \\
Densidade & $0,71 \%$ & $0,74 \%$ & $0,57 \%$ & $0,32 \%$ \\
\# de Componentes (clusters) & 91 & 83 & 110 & 182 \\
Autores no Componente Principal & $34(13,3 \%)$ & $61(19,2 \%)$ & $37(9,02 \%)$ & $260(32,3 \%)$ \\
Autores Isolados & $21(8,23 \%)$ & $11(3,47 \%)$ & $9(2,19 \%)$ & $33(4,09 \%)$ \\
\hline
\end{tabular}

Nota. Fonte: Elaborado pelos autores com base nos dados coletados.

Verifica-se, ainda, que o indicador de densidade $(\Delta \approx 0,32 \%)$ dos relacionamentos na rede global foi baixo, mas esse resultado permite supor que, da rede de Finanças no Brasil, apresenta-se quase três vezes mais densa que a de Organizações e Estratégia $(\Delta \approx 0,11 \%)$, analisada por Rossoni $\mathrm{e}$ Machado-da-Silva (2008), e a área de Gestão de Operações $(\Delta \approx 0,08 \%)$, estudada por Martins et al. (2010). Para avaliar os padrões de cooperação local, torna-se mais interessante avaliar a formação dos componentes, i.e., clusters, ou agrupamento de pesquisadores ligados entre si.

Com o aumento do tamanho da rede, o número de componentes experimentou algum aumento, embora em uma escala menor que número de autores que atuam no campo. Entre 2003 a 2006, foram formados 91 clusters; no período entre 2007 e 2009 identificou-se 83 clusters; e 110 entre 2010 e 2012 (ver Figura 3). Em outras palavras, o número de agrupamentos (novamente: componentes, ou clusters) aumentou ao longo dos três subperíodos, sugerindo algum tipo de fragmentação da rede.

O tamanho dos componentes identificados indica que, do primeiro para o segundo subperíodo analisado, observou-se aumento da proporção de autores interligados no maior cluster, i.e., o tamanho do componente principal aumentou de $13,3 \%$ para 19,2\% entre o primeiro e o segundo subperíodo. No entanto, entre o segundo e o terceiro subperíodo verifica-se uma queda substancial da parcela de autores interligados entre si no componente principal, de 19,2\% dos autores para apenas $9,02 \%$.

Essa fragmentação da rede, decorrente do aumento do número de componentes, associada à diminuição da parcela de pesquisadores no componente principal, aponta para um crescimento dos laços fracos, i.e., maior eficiência dos laços, ou aumento de Structural Holes. A título de comparação com resultados anteriormente obtidos para outros campos de conhecimento, tomando como base a literatura internacional, convém observar o que é ilustrado na Tabela 5. Tendo em vista o caráter inédito no Brasil, e ainda não ter sido encontrado qualquer trabalho na literatura internacional acerca de temática similar à que é empregada nesta pesquisa, i.e., estudo da topografia da rede de autores de Finanças, não se fez comparações com outros estudos empíricos anteriormente desenvolvidos no mesmo campo de conhecimento.

De forma consolidada, na rede do total do período analisado, o componente principal é composto por 260 autores, o que equivale a 32,3\% dos autores do campo. Segundo Newman (2004), nos campos de biologia, física e matemática no contexto internacional, o componente principal representa de $82 \%$ a 92\%; para Ciência da Computação, e essa proporção foi de 57,2\% (Newman, 2001). Dessa forma, o componente principal do Campo de Finanças no Brasil é relativamente menor que os encontrados em outros campos internacionais. 


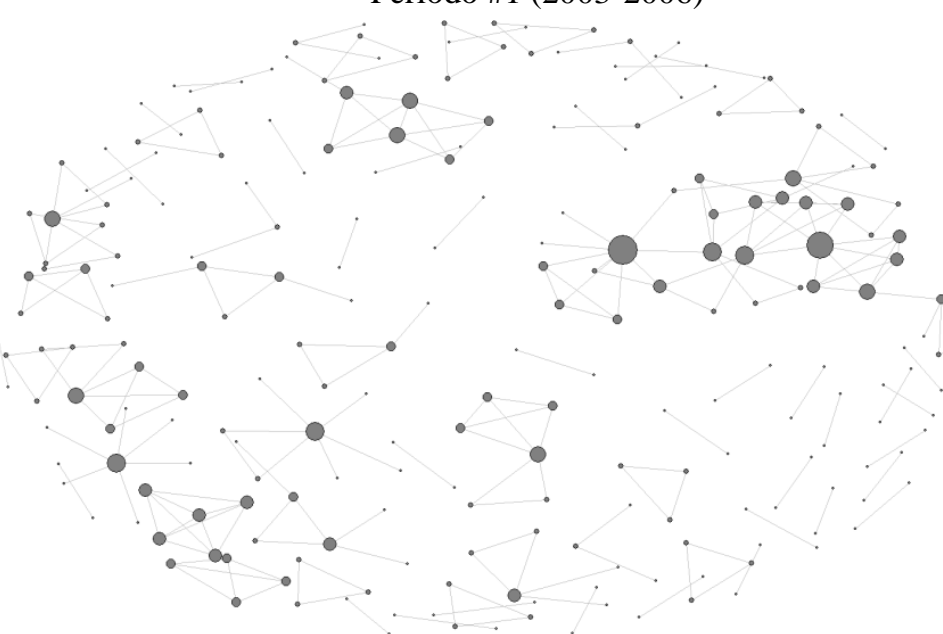

Período \#3 (2010-2012)

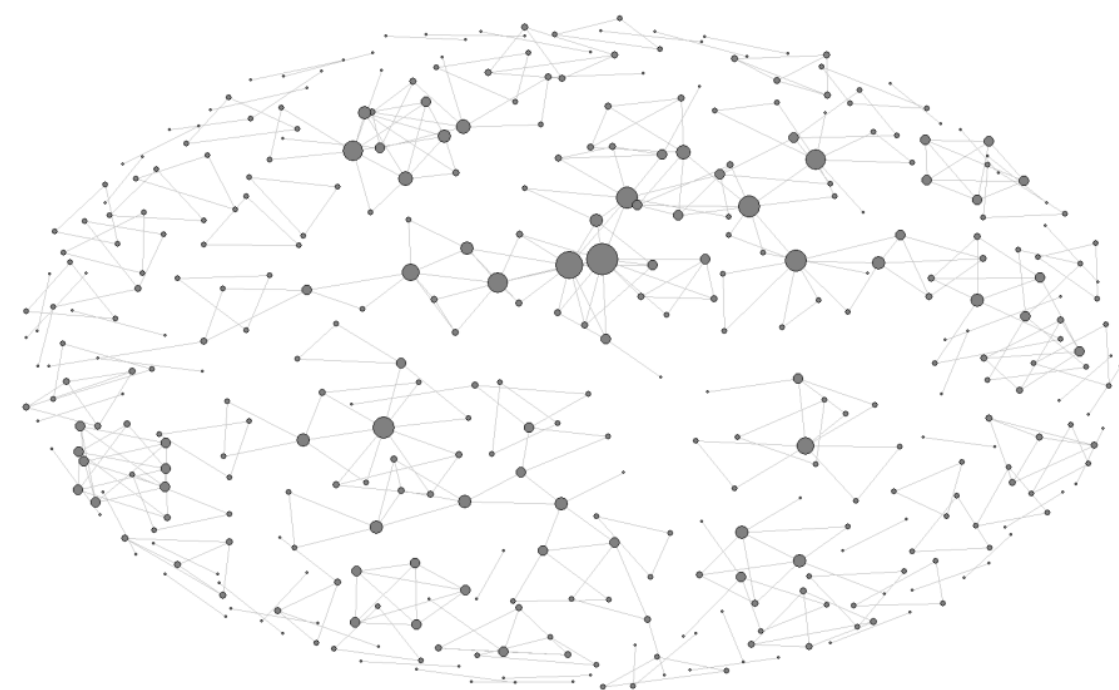

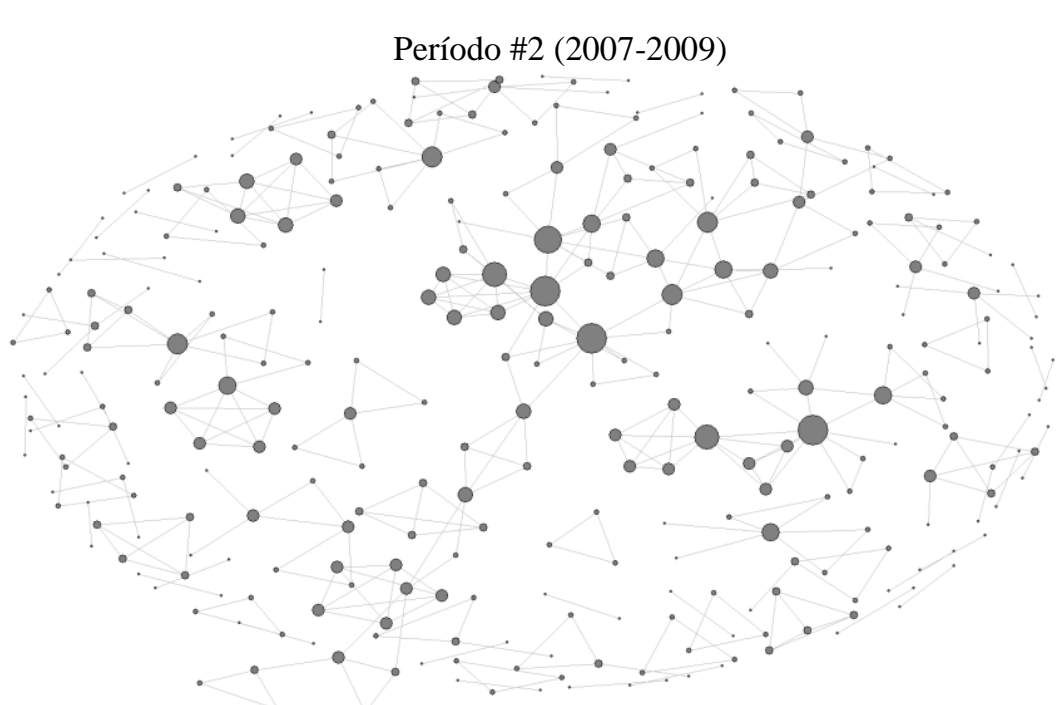

Período Completo (2003-2012)

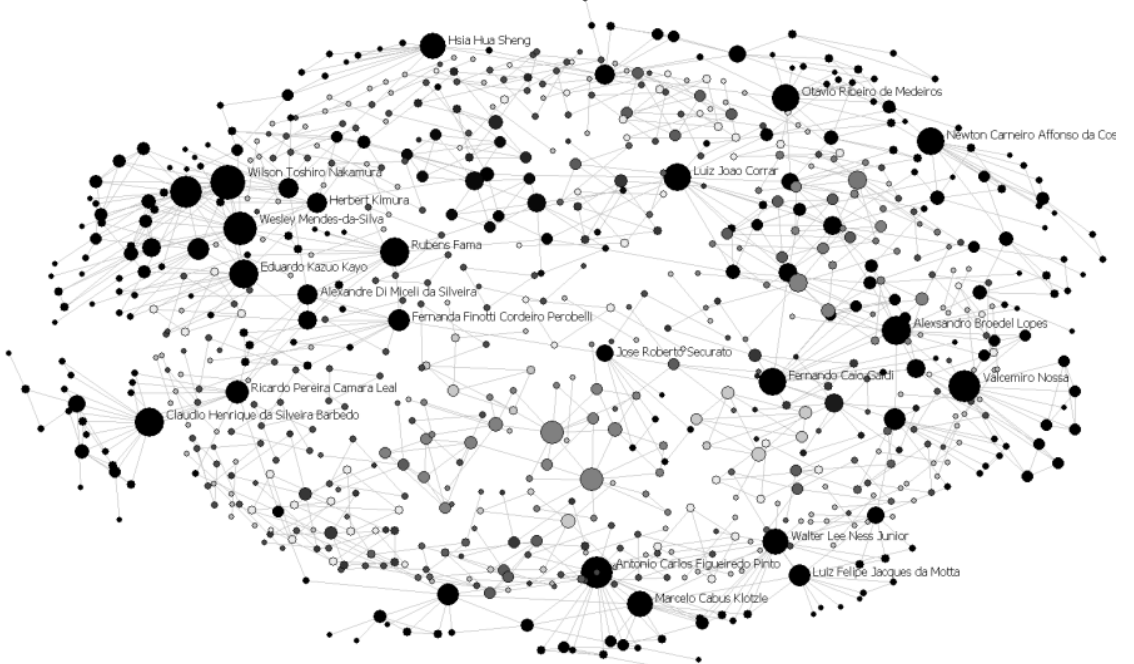

Figura 3. Evolução da Topografia da Rede de Pesquisadores em Finanças no Brasil.

Fonte: Elaborado pelos autores com base nos dados coletados. 
No Brasil, Rossoni e Machado-da-Silva (2008) encontraram que o componente principal do campo de Estratégia e Estudos Organizacionais foi de 37,9\%; em Administração da Tecnologia da Informação, o componente principal equivalia a apenas $12 \%$ dos pesquisadores do campo (Rossoni \& Hocayen-da-Silva, 2008). Portanto, embora o componente principal do campo de Finanças do Brasil seja menor que os padrões encontrados internacionalmente, este se apresenta maior que o encontrado em outras áreas da Administração no Brasil.

Tabela 5

Comparação dos Estudos em Diferentes Campos do Conhecimento

\begin{tabular}{lcccc}
\hline Campo de conhecimento & Período estudado & \# de Autores & $\begin{array}{c}\text { \#Médio de laços } \\
\text { por Autor }\end{array}$ & $\begin{array}{c}\text { \%dos Autores no } \\
\text { componente } \\
\text { principal }\end{array}$ \\
\hline $\begin{array}{l}\text { Internacional } \\
\text { Negócios }^{\mathrm{a}}\end{array}$ & $1980-2002$ & 10.176 & 2,86 & 45,4 \\
Serviços $^{\mathrm{b}}$ & $1995-2010$ & 2.457 & 2,55 & 28,4 \\
Medicina $^{\mathrm{c}}$ & $1995-1999$ & 1.520 .251 & 16,93 & 92,6 \\
Física $^{\mathrm{d}}$ & $1995-1999$ & 52.909 & 9,27 & 85,4 \\
Matemática $^{\mathrm{d}}$ & $1995-1999$ & 253.339 & 3,90 & 82,0 \\
Sociologia $^{\mathrm{e}}$ & $1963-1999$ & 197.976 & 1,88 & 34,5 \\
Brasil & $1997-2009$ & 3.148 & 2,52 & 48,5 \\
Gestão de Operações $^{\mathrm{f}}$ & $1997-2005$ & 2.072 & 2,25 & 37,9 \\
Estratégia e Organizações $^{\mathrm{g}}$ & $2003-2012$ & 806 & 2,55 & 32,26 \\
Finanças $^{\mathrm{h}}$ & & & & \\
\hline
\end{tabular}

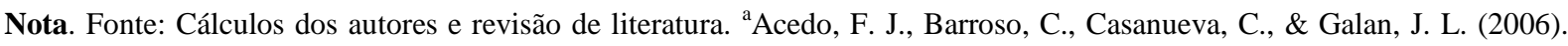
Co-authorship in management and organizational studies: an empirical and network analysis. Journal of Management

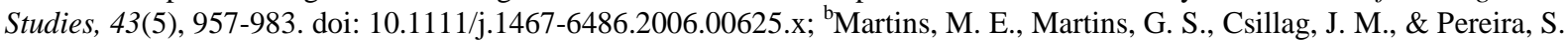
C. F. (2012). Service's scientific community: a social network analysis (1995-2010). Journal of Service Management, 23(3), 455-469. doi: 10.1108/09564231211248507; ' Newman, M. E. J. (2001). The structure of scientific collaboration networks. Proceedings of National Academic Sciences, 98(2), 404-409. doi: 10.1073/pnas.021544898; ${ }^{\mathrm{d} N e w m a n, ~ M . ~ E . ~ J . ~(2004) . ~}$ Coauthorship networks and patterns of scientific collaboration. Proceedings of the National of Academic Sciences, 101(1), 5200-5205. doi: 10.1073/pnas.0307545100; ${ }^{\mathrm{e}}$ Moody, J. (2004). The structure of a social science collaboration network: disciplinary cohesion from 1963 to 1999. American Sociological Review, 69(2), 213-238. doi:

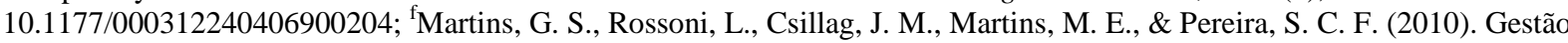
de operações no Brasil: uma análise do campo científico a partir da rede social de pesquisadores. RAE Eletrônica, 9(2). Recuperado de http://www.scielo.br/pdf/raeel/v9n2/a04v9n2.pdf. doi: 10.1590/S1676-56482010000200004; ${ }^{g}$ Rossoni, L., \& Machado-da-Silva, C. L. (2008). Análise institucional da construção do conhecimento científico em mundos pequenos. Faces, 7(1), 25-43, ${ }^{\mathrm{h}}$ Resultados desta pesquisa.

Para inferências mais aprofundadas acerca do agrupamento local dos indivíduos na rede e a estrutura da rede, utilizou-se a dinâmica de Small Worlds, empregada em estudos anteriormente desenvolvidos no campo de Finanças (Baum, Rowley, \& Shipilov, 2004). O pressuposto deste fenômeno é de que os atores, aqui chamados pesquisadores, participantes de uma rede, podem formar grupos coesos e conectar-se com outros grupos a partir de um pequeno número de intermediários (Newman, 2004; Newman, Strogatz, \& Watts, 2001; Watts \& Strogatz, 1998).

$\mathrm{Na}$ Tabela 6, foram calculados os indicadores necessários para tal análise, e estes foram confrontados com os dados aleatórios, que correspondem aos indicadores elaborados conforme Watts e Strogatz (1998). Percebe-se que a configuração da rede de pesquisadores no campo de Finanças no Brasil parece seguir a lógica da dinâmica de Small Worlds ao longo período estudado, já que $\Theta \gg 1$. Apesar de a densidade global da rede ser baixa (i.e., há pouca relação entre os autores em nível global) 
e os pesquisadores possuírem forte agrupamento local $\left(C_{\Delta_{\text {observado }}}\right)$, em valores maiores que a expectativa, segundo modelo de Newman (2001), a distância média ( $L_{\text {observada }}$ ) foi maior que o esperado em todos os subperíodos, bem como no total de todos os períodos (2003-2012). Em termos de distância entre os pesquisadores pertencentes à rede composta pelos membros do componente principal (o cluster empregado para obtenção das métricas necessárias à verificação da existência de mundo pequeno), a Tabela 6 indica a diminuição da distância observada, ao longo do período ( 4 no primeiro, para $\sim 3,3$ no terceiro subperíodo).

Tabela 6

Evolução da Estatística de Small Worlds na Rede de Pesquisadores em Finanças no Brasil

\begin{tabular}{|c|c|c|c|c|}
\hline \multirow[b]{3}{*}{ Parâmetros para diagnóstico de Small World } & \multirow{2}{*}{\multicolumn{4}{|c|}{ Período analisado }} \\
\hline & & & & \\
\hline & 2003-06 & 2007-09 & 2010-12 & 2003-12 \\
\hline \multicolumn{5}{|l|}{ Painel A: Parâmetros observados na rede } \\
\hline Densidade $(\Delta)$ & 0,102 & 0,060 & 0,105 & 0,015 \\
\hline \# de Autores $(n)$ & 34 & 61 & 37 & 260 \\
\hline Média de laços constituídos por Autor $(k)$ & 3,353 & 3,607 & 3,784 & 3,769 \\
\hline$C_{\triangle \text { observado: }}$ Coeficiente de Agrupamento observado & 0,782 & 0,747 & 0,744 & 0,743 \\
\hline$L_{\text {observado }}$ : Distância Média observada & 3,998 & 4,407 & 3,332 & 5,698 \\
\hline Distância Máxima (Diâmetro) & 9 & 11 & 9 & 13 \\
\hline \multicolumn{5}{|l|}{ Painel B: Parâmetros esperados (simulados) para a rede } \\
\hline$C_{\Delta \text { esperado }}:$ Coeficiente de Agrupamento esperado $(k / n)$ & 0,0986 & 0,0591 & 0,1023 & 0,014 \\
\hline$L_{\text {esperado }}$ : Distância Média Esperada $(\ln (n) / \ln (k))$ & 2,915 & 3,204 & 2,713 & 4,191 \\
\hline \multicolumn{5}{|l|}{ Painel C: Cálculo da estatística de Small Worlds $(\Theta)$} \\
\hline \multicolumn{5}{|l|}{ Indicadores de small world } \\
\hline$C_{\Delta \text { observado }} / L_{\text {observado }}$ & 0,20 & 0,17 & 0,22 & 0,130 \\
\hline$L_{\text {esperado }} / C_{\Delta \text { esperado }}$ & 29,56 & 54,19 & 26,53 & 289,112 \\
\hline$\Theta=\left[C_{\Delta \text { observado }} / L_{\text {observado }}\right] .\left[L_{\text {esperado }} / C_{\Delta \text { esperado }}\right]$ & 5,78 & 9,19 & 5,92 & 37,699 \\
\hline
\end{tabular}

Nota. Fonte: Elaborado pelos autores com base nos dados coletados. Os cálculos são válidos para o componente principal das redes, conforme Newman, M. E. J. (2001). The structure of scientific collaboration networks. Proceedings of National Academic Sciences, 98(2), 404-409. doi: 10.1073/pnas.021544898 e Moody, J. (2004). The structure of a social science collaboration network: disciplinary cohesion from 1963 to 1999. American Sociological Review, 69(2), 213-238. doi: 10.1177/000312240406900204. Valores da estatística $\Theta \gg 1$ indicam a existência de Small Worlds. As métricas reportadas nesta tabela são referentes à configuração do componente principal da rede composta pelos pesquisadores em cada período.

Ao mesmo tempo, o coeficiente de agrupamento observado na rede do componente principal mostrou redução ao longo do período, tal como apresenta a Figura 4. Como resultado do comportamento conjunto da distância entre os pesquisadores pertencentes à rede e o coeficiente de agrupamento desses, tal como prescrevem Newman et al. (2001), a estatística de Small Worlds $(\Theta)$ apresentou valores maiores que 1, indicando a existência de Small Worlds na rede de pesquisadores de Finanças no Brasil, em linha com o critério indicado por Watts e Strogatz (1998). Em que pese o número de autores e de autorias ter aumentado, e a densidade ser relativamente baixa (mas não se comparada a outros campos de conhecimento no Brasil), se considerado o componente principal, existem indícios da existência de Small Worlds. 


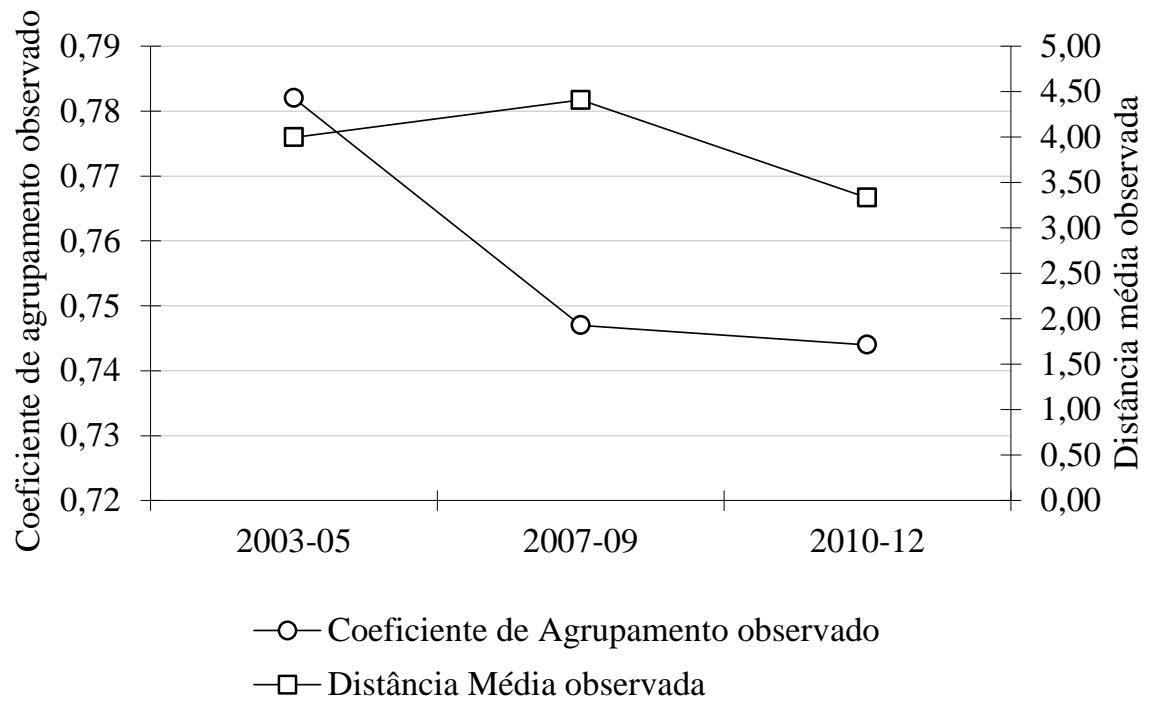

Figura 4. Evolução das Métricas de Small Worlds na Rede de Pesquisadores de Finanças no Brasil. Fonte: Cálculos dos autores com base nos dados coletados. Este gráfico apresenta a evolução das métricas de small worlds verificas na rede de pesquisadores no campo de finanças no Brasil, nota-se o crescimento da distância entre os pesquisadores, e o aumento do coeficiente de agrupamento entre eles, ao longo do período analisado (2003-2012).

E esses indícios serão tão fortes quanto maiores forem: a estatística de Small Worlds (ver Tabela 6) e o percentual dos membros da rede global no seu componente principal (examinando a Tabela 5 verifica-se que o campo de Finanças apresenta a menor parcela, se comparada a Estratégia e a Gestão de Operações), i.e., o cluster com maior número de atores. Nesse sentido, o presente estudo aponta para a existência de Small Worlds na comunidade de Finanças. Contudo, quando comparada a estudos brasileiros anteriormente realizados, o valor de $\Theta$ que foi aqui encontrado ao redor de 37,7, pode ser considerado pouco expressivo, quando comparado ao encontrado por Martins et al. (2010), no campo de Gestão de Operações, que foi de 209,5.

\section{Centralidade dos pesquisadores}

Nesta subseção, foram identificados os atores mais centrais na rede de pesquisadores do campo de Finanças no Brasil. A centralidade foi discutida tanto em termos do número de laços, em primeiro nível, estabelecidos por um pesquisador (centralidade de grau, i.e., Degree), como também na capacidade de o pesquisador posicionar-se como um intermediador de relações entre outros participantes da rede (centralidade de intermediação, i.e. Betweenness). São apresentados e discutidos, ainda, os coeficientes estimados, via regressão linear OLS, a respeito da associação de tais medidas de centralidade, bem como eficiência dos laços (Structural Holes), com o volume de produção científica dos pesquisadores. A Tabela 7 apresenta os autores mais centrais, em ambos os conceitos. Com relação à centralidade de grau, Nakamura, W. T. - Mackenzie; Mendes-Da-Silva, W. - FGV/EAESP se destacam. Cada um deles estabeleceu 17 e 16 laços diretos, cada um na produção de seus artigos.

A outra proxy de centralidade utilizada - a centralidade de intermediação que avalia a capacidade do autor de participar de grupos diferentes em uma mesma rede e também pode indicar aqueles atores que controlam o fluxo de informação na rede. Neste sentido, Leal, R. P. C. Coppead/UFRJ e Famá, R.- PUC/SP se destacam. Alguns autores, como Famá, R., Leal, R. P. C., Lopes, A. B., Mendes-Da-Silva, W. e Galdi, F. C. combinam alta centralidade de grau de intermediação. Outros que não apareciam com grande centralidade de grau, apresentaram-se com grande capacidade de intermediar relações na rede, como, por exemplo, os pesquisadores Silveira, A. D. M. e Barros, L. A. B. C., ambos FEA/USP, que se apresentam como alguns dos mais centrais da rede, segundo esta métrica de centralidade. 
Tabela 7

Autores com Maior Centralidade de Grau e de Intermediação no Período 2003-2012

\begin{tabular}{|c|c|c|c|c|}
\hline$\#$ & Pesquisador & Degree & Pesquisador & Betweeness \\
\hline 1 & Nakamura, W.T. ${ }^{\dagger \dagger}$ & 17 & Leal, R.P.C.† & $9.303,019$ \\
\hline 2 & Mendes-da-Silva, W. ${ }^{\dagger}$ & 16 & Fama, R..$^{\dagger}$ & $8.019,207$ \\
\hline 3 & Pinto, A.C.F. & 15 & Silveira, A.D.M. $^{\dagger}$ & $6.874,757$ \\
\hline 4 & Martin, D.M.L. ${ }^{\dagger}$ & 15 & Lopes, A.B. & $5.936,951$ \\
\hline 5 & Nossa, V..$^{\dagger}$ & 15 & Corrar, L.J. ${ }^{\dagger}$ & $5.915,294$ \\
\hline 6 & Lopes, A.B. & 14 & Funchal, B. & $5.430,644$ \\
\hline 7 & Barbedo, C.H.S. $^{\dagger}$ & 14 & Galdi, F.C. & $4.857,947$ \\
\hline 8 & Kayo, E.K." & 14 & Brandao, L.E.T ${ }^{\ddagger}$ & $4.855,336$ \\
\hline 9 & Fama, R..$^{\dagger}$ & 14 & Saito, R. ${ }^{\star \dagger}$ & $4.849,237$ \\
\hline 10 & Galdi, F.C. & 13 & Sheng, H.H. ${ }^{\dagger}$ & $4.704,026$ \\
\hline 11 & Corrar, L.J. ${ }^{\ddagger}$ & 13 & Lemme,C.F. & $4.427,349$ \\
\hline 12 & Costa Junior, N.C.A. ${ }^{\sharp \dagger}$ & 13 & Barros, L.A.B.C. & $4.393,025$ \\
\hline 13 & Medeiros, O.R. ${ }^{\dagger \dagger}$ & 13 & Eid Junior, W. & $4.360,736$ \\
\hline 14 & Sheng, H.H. ${ }^{\dagger}$ & 12 & Barbedo, C.H.S. ${ }^{\dagger}$ & $4.207,000$ \\
\hline 15 & Klotzle, M.C. & 12 & Mendes-da-Silva, W..$^{\dagger}$ & $4.066,447$ \\
\hline 16 & Junior, W.L.N. $^{\dagger}$ & 12 & Fortunato, G.X. & $4.032,814$ \\
\hline 17 & Bressan, A.A. ${ }^{\dagger}$ & 11 & Perobelli, F.F.C. $^{\dagger}$ & $4.008,426$ \\
\hline 18 & Amaral, H.F. ${ }^{+\dagger}$ & 11 & Medeiros, O.R. ${ }^{\ddagger \dagger}$ & $3.790,647$ \\
\hline 19 & Leal, R.P.C. ${ }^{\dagger}$ & 11 & Securato, J.R..$^{\dagger}$ & $3.659,616$ \\
\hline 20 & Funchal, B. & 10 & Capelletto, L.R. & $3.587,499$ \\
\hline
\end{tabular}

Nota. Fonte: Cálculos dos autores a partir dos dados coletados. $\$$ Pesquisador bolsista de produtividade pelo CNPq. $†$ Pesquisador classificado como Continuante, mediante critérios propostos por Braun, T., Glänzel, W., \& Schubert, A. (2001). Publication and cooperation patterns of the authors of neuroscience journals. Scientometrics, 50(3), 499-510. doi: 10.1023/A:1012774206340

Em outras palavras, estes pesquisadores têm um posicionamento central na rede tal que lhes permite intermediar um elevado número de relações, mesmo que não as tenham em quantidade de forma direta. Outros pesquisadores, que possuem alta centralidade de grau e intermediação, combinam as duas características: possuem muitos relacionamentos diretos e outras relações indiretas se originam destes. A Tabela 8 apresenta os valores médios obtidos para diferentes métricas de posicionamento dos pesquisadores no âmbito da rede, distribuídos pelas suas categorias, i.e., mediante critério proposto por Braun et al. (2001), em termos de regularidade e volume de produção científica publicada em revistas brasileiras. Nota-se que $76 \%$ dos autores publicaram apenas um trabalho na década estudada. Adicionalmente, uma parcela de apenas 3\% dos pesquisadores considerados apresentou publicação julgada regular nos últimos anos. Ou melhor, os pesquisadores Continuantes apresentaram uma centralidade de grau média próxima de 11 laços, e uma produção média ao redor de 8 artigos.

Tendo em vista resultados obtidos em estudos anteriores, tais como Lee e Bozeman (2005), existe a expectativa de que métricas de centralidade e produção estejam associadas de forma significante. Assim, os autores Continuantes, na maioria das oportunidades, são responsáveis por constituir parcerias de pesquisa cujo resultado é o acúmulo de vantagens cumulativas. Dito de outra 
forma, os Continuantes assumem papel essencial no campo de conhecimento, já que, sendo atores mais centrais, tendem a possuir maior poder de difusão e de legitimação do conhecimento acumulado no campo de conhecimento, em decorrência de seu capital social, conforme argumentação apresentada por Zucker e Darby (1996).

Tabela 8

Média de Indicadores de Posicionamento na Rede de Pesquisadores (por Categoria de Pesquisadores)

\begin{tabular}{rccccc}
\hline Categoria do pesquisador & \# de Pesquisadores (\%) & Degree & \#de Artigos & Beetweeness & Structuralholes \\
\hline One Timer & $610(76)$ & 1,80 & 1,00 & 0,00 & 0,41 \\
Entrante & $29(4)$ & 3,45 & 2,34 & 509,20 & 0,41 \\
Transiente & $85(11)$ & 4,62 & 3,28 & 591,97 & 0,52 \\
Retirante & $56(7)$ & 3,21 & 2,54 & 277,72 & 0,46 \\
Continuante & $26(3)$ & 11,08 & 8,23 & 3032,79 & 0,72 \\
\hline Total & $806(100)$ & 2,55 & 1,63 & 197,88 & 0,43 \\
\hline
\end{tabular}

Nota. Fonte: Cálculos dos autores com base nos dados coletados. Para classificação dos pesquisadores foram empregados os procedimentos indicados por Braun, T., Glänzel, W., \& Schubert, A. (2001). Publication and cooperation patterns of the authors of neuroscience journals. Scientometrics, 50(3), 499-510. doi: 10.1023/A:1012774206340

A respeito das associações entre produção científica e posicionamento do pesquisador na rede, foram conduzidas regressões lineares via procedimento OLS, com variável dependente sendo o número de artigos publicados pelo $i$-ésimo pesquisador ao longo de todo o período estudado. As variáveis independentes foram as métricas de centralidade de grau (Degree) e de intermediação (Betweenness), além da eficiência dos laços constituídos pelo pesquisador, cuja proxy é Structural Holes, i.e., proporção dos laços não redundantes que o pesquisador constituiu ao compartilhar autorias de trabalhos publicados nas revistas científicas consideradas nesta pesquisa.

A Tabela 9 apresenta os coeficientes obtidos na simulação do modelo (4). As métricas de fit do modelo foram escolhidas mediante proposta de Vartanian, Cia e Mendes-da-Silva (2013). Assim, foi verificada a existência de heterocedasticidade por meio do teste de Breusch-Pagan, cuja hipótese nula, i.e., inexistência de heterocedasticidade foi rejeitada $(\mathrm{LM}=1779,66 ; \mathrm{p}<0,01)$. A partir disso, optou pelos erros-padrão robustos à heterocedasticidade (variante $\mathrm{HC} 1$ ). Ainda no que se refere às métricas de fit do modelo, convém ainda destacar que não foram encontrados indícios de multicolinearidade entre essas variáveis independentes, via Fator de Inflação da Variância (VIF), conforme estabelecem Tabachnik e Fidell (2007).

Tabela 9

Efeito Marginal do Posicionamento do Pesquisador na Rede sobre sua Produção

\begin{tabular}{lccccc}
\hline & Coeficiente & Erro Padrão & $\mathrm{t}$ & VIF & \\
\hline const & $-0,887$ & 0,167 & $-5,309$ & & $* * *$ \\
Centralidade de grau (Degree) & 0,488 & 0,038 & 12,916 & 2,032 & $* * *$ \\
$\begin{array}{l}\text { Centralidade de intermediação } \\
\begin{array}{l}\text { (Beetweeness) } \\
\text { Eficiência dos laços (Structural holes) }\end{array}\end{array}$ & $2,92.10^{-4}$ & $1,188.10^{-4}$ & 2,453 & 2,243 & $* *$ \\
\hline
\end{tabular}


Tabela 9 (continuação)

\begin{tabular}{lcccc}
\hline & Coeficiente & Erro Padrão & t & VIF \\
\hline R-quadrado Ajustado & 0,804886 & & & \\
$\mathrm{~F}(3,802)$ & 137,7953 & $* * *$ & \\
Critério de Akaike & 1777,173 & & \\
$\mathrm{~N}$ & 806 & & \\
\hline
\end{tabular}

Nota. Fonte: Cálculos dos autores com base nos dados coletados. Entre parênteses está o erro-padrão da estimativa de cada coeficiente. Não foram encontrados indícios de multicolinearidade entre as variáveis independentes, mediante procedimento do VIF, já que não foram observados valores próximos de 10. Variável dependente = número de artigos publicados no período de 2003 a 2012. A verificação de existência a priori de heteroscedasticidade foi verificada com emprego do Teste de Breusch-Pagan, para o qual foi rejeitada a hipótese nula de inexistência de heteroscedasticidade $(\mathrm{LM}=1779,66 ; \mathrm{p}<0,01)$. Portanto, foi utilizada estimativa por erros-padrão robustos à heteroscedasticidade (variante HC1). Os parâmetros estimados para os coeficientes sugerem que os autores de maior centralidade e maior eficiência de laços tenderam a apresentar maior número de artigos publicados no período estudado (2003-2012). $* * *$ p-value $<0,01$.

A respeito das estimativas dos coeficientes do modelo (4), constata-se que, em média, os autores de maior centralidade de grau $\left(\widehat{\beta_{1}} \approx 0,488 ; p<0,01\right)$ e de intermediação $\widehat{\beta_{2}} \approx 2,92.10^{-4}$ (; $p<0,05)$, assim como maior eficiência de laços $\left(\widehat{\beta_{3}} \approx 2,797 ; p<0,01\right)$, obtiveram maior número de artigos publicados. Dito de outra forma, conforme argumentos defendidos por Acedo et al. (2006), e ratificados por Martins, Martins, Csillag e Pereira (2012), os quais assumem que pesquisadores de maior prestígio social, medido pela sua centralidade no âmbito da rede de autores, alcançam produção científica mais expressiva. Adicionalmente, entende-se que, conforme Merton (1973), Kuhn (1978), Pepe (2010) e Lemarchand (2012), o conhecimento é socialmente construído. E, nesse sentido, o entendimento da estrutura e da dinâmica da colaboração na pesquisa científica em Finanças parece exercer papel preponderante na promoção do amadurecimento do campo de conhecimento.

\section{Considerações Finais}

Este trabalho teve como objetivo analisar as propriedades estruturais das redes de relações entre os pesquisadores na área de Finanças no Brasil, no período 2003-2012. Assim, com base em dados pertencentes a 532 artigos publicados por 806 diferentes pesquisadores, que em sua maioria $(\sim 76 \%)$ publicaram apenas um único artigo nesse período, os resultados empíricos obtidos sugerem três resultados principais.

Primeiro, quanto maior a visibilidade do pesquisador no campo de conhecimento, medida por duas métricas de centralidade, i.e., Degree e Betweenness, juntamente com maior eficiência de laços, medida por Structural Holes, maior foi a quantidade de artigos publicados. Em outras palavras: aqueles autores com maior centralidade e menor redundância de laços, em média, obtiveram maior número de artigos publicados em periódicos selecionados no Brasil, considerando o período em tela.

O segundo resultado principal indica que o campo de Finanças no Brasil apresenta configuração estrutural de rede tal que não se pode excluir a possibilidade da existência de Small Worlds no âmbito da rede de pesquisadores. E, em terceiro lugar, mas não menos relevante, o campo de Finanças tem crescido em número de pesquisadores e número de trabalhos publicados, mas especialmente no que se refere ao número de pesquisadores no campo, o que reforça a suposição de que se pode classificar o campo de Finanças como ainda pouco maduro, conforme discutem Nooy et al. (2005).

A presente pesquisa possui sua relevância principalmente apoiada na sua capacidade de, pioneiramente no Brasil, evidenciar a evolução da estrutura da rede de pesquisadores de Finanças na última década, por meio do emprego de metodologia até então não empregada - ARS, a qual 
possibilita entender aspectos estruturais da rede de pesquisadores, bem como o posicionamento dos atores. Essa questão está em linha com recomendações e sugestões anotadas em recente trabalho desenvolvido por Leal et al. (2013), quando esses citam redes de pesquisadores como proposta de caminhos para a expansão das fronteiras do estudo de Finanças no Brasil.

Os resultados revelaram que apenas uma pequena parcela $(\sim 3 \%)$ dos pesquisadores possui publicação regular nos periódicos nacionais, quando considerada a última década. A rede de pesquisadores de Finanças possui densidade global expressivamente maior que a encontrada em outras áreas, como Gestão de Operações e Estratégia. Contudo, em paralelo, foi constatado o crescimento do número de clusters de autores, o que se reflete na baixa parcela de pesquisadores participantes da rede de maior número de pessoas ( $32 \%$ dos pesquisadores). Em que pese o nível de fragmentação da rede, quando considerado o maior conjunto de autores conectados, foram verificadas as propriedades de Small Worlds. Isso sugere que os pesquisadores estão arranjados em grupos menores, interconectados por pesquisadores com elevado poder de intermediação de relações.

O trabalho indica ainda um movimento de abertura da rede de coautores, significando que a tarefa de construção de artigos segue cada vez mais a afirmativa de Castells e Cardoso (2005) e Nohria e Ecles (1992), segundo a qual a forma de organização dos campos de conhecimento, sendo parte da sociedade, constitui rede. Como consequência disso, torna-se paulatinamente mais evidente a necessidade de interação com públicos externos (sejam indivíduos ou instituições) para construção de conhecimento, com o mínimo de esforço redundante, i.e., aproveitando as oportunidades de compartilhamento de competências e habilidades.

Na perspectiva prática, ou gerencial, de que o trabalho é importante, já que indica caminhos de reflexão e ações das instituições que abrigam os autores, uma delas seria analisar especificamente no seu grupo de pesquisadores qual a situação de rede dominante e relacionar os dados com a produção de artigos científicos. Outra ação gerencial, considerando que a maioria dos autores tem baixa produção no período considerado, seria a instituição incentivar os ex-alunos a continuarem investigando e escrevendo sobre o assunto, indo além do ritual de escrever um artigo que é o resumo de sua dissertação ou de sua tese.

Uma possível terceira ação gerencial das instituições seria realizar um trabalho (com fóruns, intercâmbios e outras ações de aproximação) de incentivo à produção interorganizações, uma vez que os dados mostraram tendência de clusters definidos pela região e pela instituição. Finalmente, para a comunidade científica e os reitores de programas de graduação e pós-graduação, o trabalho pode servir de modelo para a investigação de outros campos de conhecimentos, utilizando essa perspectiva das afirmativas sobre redes sociais.

O presente trabalho possui limitações que são decorrentes das escolhas em termos de desenho de pesquisa, tais como - período de tempo estudado, revistas consideradas no exame de literatura brasileira em finanças, desconsideração de trabalhos publicados em revistas editadas em outros países. Mas, em que pese a existência de limitações decorrentes do desenho da presente pesquisa, os resultados alcançados neste trabalho contribuem para o melhor entendimento da dinâmica de colaboração entre os pesquisadores de Finanças no Brasil, e aponta para um campo de pesquisa ainda pouco explorado no país.

O campo de Finanças no Brasil ainda pode ser visto como pouco maduro, tendo em vista o explícito crescimento obtido nos últimos anos, ao menos se considerados os argumentos de Nooy, et al. (2005). Assim, a título de sugestões de pesquisas futuras, pode-se apontar: (a) fazer estudo similar considerando a produção internacional; (b) buscar entender os determinantes do estabelecimento de parcerias de pesquisa por meio de survey dirigido aos pesquisadores; (c) conforme Chan, Chen e Fung (2009), verificar a existência dos efeitos pedigree e placement na produção científica brasileira no campo de Finanças. 


\section{Agradecimento}

Este trabalho foi produzido em colaboração com a AES Eletropaulo (Projeto \#4690000854), mediante projeto de P\&D, com foco em inovação financeira. Os autores são sinceramente gratos aos revisores anônimos da RAC, pelas sugestões e críticas que contribuíram para melhorar o manuscrito original deste trabalho.

\section{Referências}

Acedo, F. J., Barroso, C., Casanueva, C., \& Galan, J. L. (2006). Co-authorship in management and organizational studies: an empirical and network analysis. Journal of Management Studies, 43(5), 957-983. doi: 10.1111/j.1467-6486.2006.00625.x

Barabási, A. L., Jeong, H., Néda, Z., Ravasz, E., Schubert, A., \& Vicsek, T. (2002). Evolution of the social network of scientific collaborations. Physica A, 311(3/4), 590-614. doi: 10.1016/S03784371(02)00736-7

Baum, J. A. C., Rowley, T. J., \& Shipilov, A. V. (2004). The small world of Canadian capital markets: statistical mechanics of investment bank syndicate networks, 1952-1989. Canadian Journal of Administration Sciences, 21(4), 307-325. doi: 10.1111/j.1936-4490.2004.tb00347.x

Borgatti, S. (1997). Structural holes: unpacking Burt's redundancy measures. Connections, 20(1), 35-38.

Braun, T., Glänzel, W., \& Schubert, A. (2001). Publication and cooperation patterns of the authors of neuroscience journals. Scientometrics, 50(3), 499-510. doi: 10.1023/A:1012774206340

Burt, R. (1992). Structural holes: the social structure of competition. Cambridge: Harvard University Press.

Castells, M., \& Cardoso, G. (2005). The network society: from knowledge to policy. Washington: John Hopkins.

Chan, K. C., Chen, C. R., \& Fung, H.-G. (2009). Pedigree or placement? An analysis of research productivity in finance. The Financial Review, 44(1), 87-111. doi: 10.1111/j.15406288.2008.00211.x

Chan, K. C., Chen, C. R., \& Lung P. P. (2007). One-and-a-half decades of global research output in finance: 1990-2004. Rev Quant Finance Account, 28(4), 417-439. doi: 10.1007/s11156-0070018-y

Chan, K. C., Chen, C. R., \& Steiner, T. L. (2004). Who is publishing? An analysis of finance research productivity in the European region. Journal of Business Finance \& Accounting, 31(3/4), 401437. doi: 10.1111/j.0306-686X.2004.00545.x

Chassagnon, V., \& Audran, M. (2011). The impact of interpersonal networks on the innovativeness of inventors: from theory to empirical evidence. International Journal of Innovation Management, 15(5), 931-958. doi: 10.1142/S1363919611003349

Coleman, J. (1990). Foundations of social theory. Chicago: University of Chicago Press.

Davis, G. F., Yoo, M., \& Baker, W. E. (2003). The small world of corporate elite, 1982-2001. Strategic Organization, 1(3), 301-326. doi: 10.1177/14761270030013002

Freeman, L. C. (1978). Centrality in social networks conceptual clarification. Social Networks, 1(3), 215-239. doi: 10.1016/0378-8733(78)90021-7 
Goyal, S., Van der Leij, M., \& Moraga-González, J. L. (2004). Economics: an emerging small world? [Working Paper Series $N^{o}$ 1287]. CESifo. Recuperado de http://www.cesifogroup.de/DocDL/cesifo1_wp1287.pdf

Granovetter, M. (1973). The strength of weak ties. American Journal of Sociology, 78(6), 1361-1380.

Granovetter, M. (1985). Economic action and social structure: the problem of embeddedness. American Journal of Sociology, 91(3), 481-510.

Gulati, R., \& Gargiulo, M. (1999). Where do interorganizational networks come from? The American Journal of Sociology, 104(5), 1439-1493.

Hardin, W. G., III, Liano, K., Chan, K. C., \& Fok, R. C. W. (2008). Finance editorial board membership and research productivity. Review of Quantitative Finance and Accounting, 31(3), 225-240. doi: 10.1007/s11156-007-0067-2

Knoke, D., \& Kuklinski, J. H. (1982). Network analysis. Beverly Hills, CA: Sage.

Kogut, B., \& Walker, G. (2001). The small world of Germany and the durability of national networks. American Sociological Review, 66(3), 317-335. doi: 10.2307/3088882

Kuhn, T. (1978). A estrutura das revoluções científicas (2a ed.). São Paulo: Perspectiva.

Leal, R. P. C., Almeida, V. S. de, \& Bortolon, P. M. (2013). Produção científica brasileira em finanças no período 2000-2010. Revista de Administração de Empresas, 53(1), 46-55. doi: $10.1590 / \mathrm{S} 0034-75902013000100005$

Lee, S., \& Bozeman, B. (2005). The impact of research collaboration on scientific productivity. Social Studies of Science, 35(5), 673-702. doi: 10.1177/0306312705052359

Lemarchand, G. A. (2012). The long-term dynamics of co-authorship scientific networks: iberoamerican countries (1973-2010). Research Policy, 41(2), 291-305.

Lin, N. (2001). Social capital: a theory of social structure and action. Cambridge: Cambridge University Press.

Martins, G. S., Rossoni, L., Csillag, J. M., Martins, M. E., \& Pereira, S. C. F. (2010). Gestão de operações no Brasil: uma análise do campo científico a partir da rede social de pesquisadores. RAE Eletrônica, 9(2). Recuperado de http://www.scielo.br/pdf/raeel/v9n2/a04v9n2.pdf. doi: $10.1590 / \mathrm{S} 1676-56482010000200004$

Martins, M. E., Martins, G. S., Csillag, J. M., \& Pereira, S. C. F. (2012). Service's scientific community: a social network analysis (1995-2010). Journal of Service Management, 23(3), 455-469. doi: 10.1108/09564231211248507

Merton, R. (1973). The sociology of science. Chicago: University of Chicago Press.

Mizruchi, M. S. (1996). What do interlocks do? An analysis, critique, and assessment of research on interlocking directorates. Annual Review of Sociology, 22(1), 271-298. doi: 10.1146/annurev.soc.22.1.271

Moed, H. F., Glänzel, W., \& Schubert, A. (2005). Analysing scientific networks through coauthorship. In H. F. Moed, W. Glänzel, \& A. Schubert (Eds.), Handbook of quantitative science and technology research (pp. 257-276). Dordrecht: Klumer Academic Publishers.

Moody, J. (2004). The structure of a social science collaboration network: disciplinary cohesion from 1963 to 1999. American Sociological Review, 69(2), 213-238. doi: $10.1177 / 000312240406900204$ 
Newman, M. E. J. (2001). The structure of scientific collaboration networks. Proceedings of National Academic Sciences, 98(2), 404-409. doi: 10.1073/pnas.021544898

Newman, M. E. J. (2004). Coauthorship networks and patterns of scientific collaboration. Proceedings of the National of Academic Sciences, 101(1), 5200-5205. doi: 10.1073/pnas.0307545100

Newman, M. E. J., Strogatz, S. H., \& Watts, D. J. (2001). Random graphs with arbitrary degree distributions and their applications. Physical Review E - PHYS REV E., 64(2), 026118-1026118-17. doi: 10.1103/PhysRevE.64.026118

Nohria, N., \& Ecles, R. (1992). Networks and organizations: structure, form, and action. Boston: Harvard Business School.

Nooy, W. de, Mrvar, A., \& Batagelj, V. (2005). Exploratory social network analysis with pajek. New York: Cambridge University Press.

Oltheten, E., Theoharakis, V., \& Travlos, N. G. (2005). Faculty perceptions and readership patterns of finance journals: a global view. Journal of Financial and Quantitative Analysis, 40(1), 223-239.

Pepe, A. (2010). Structure and evolution of scientific collaboration networks in a modern research collaboratory (Dissertation of $\mathrm{PhD}$ ). Harvard University. Recuperado de http://dx.doi.org/10.2139/ssrn.1616935

Pitts, F. R. (1979). The Medieval river trade network of Russia revisited. Social Networks, 1(3), 285-292.

Rossoni, L., \& Hocayen-da-Silva, A. J. (2008). Cooperação entre pesquisadores da área de administração da informação: evidências estruturais de fragmentação das relações no campo científico. Revista de Administração, 43(2), 138-151.

Rossoni, L., \& Machado-da-Silva, C. L. (2008). Análise institucional da construção do conhecimento científico em mundos pequenos. Faces, 7(1), 25-43.

Scott, J. (2000). Social network analysis: a handbook (2nd ed.). London: Sage Publications.

Tabachnik, B. G., \& Fidell, L. S. (2007). Using multivariate statistics (5th ed.). Pearson: Boston.

Uzzi, B., \& Spiro, J. (2005). Collaboration and creativity: the small world problem. American Journal of Sociology, 111(2), 447-504. doi: 10.1086/432782

Vartanian, P. R., Cia, J. C., \& Mendes-da-Silva, W. (2013). Econometria: análise de dados com regressão linear. São Paulo: Saint Paul.

Wasserman, S., \& Faust, K. (1994). Social network analysis: methods and applications. Cambridge: Cambridge University Press.

Watts, D. (1999). Small worlds: the dynamics of networks between order and randomness. Princeton: Princeton University Press.

Watts, D., \& Strogatz, S. (1998). Collective dynamics of "small-world" networks. Nature, 393, 440442. doi: $10.1038 / 30918$

Zucker, J. G., \& Darby, M. R. (1996). Star scientists and institutional transformation: patterns of invention and innovation in the formation of the biotechnology industry. Proceedings of the National Academy of Sciences of the United States of America, 93(23), 12709-12719. 